\title{
Heart Rate Variability and Cardiopulmonary Dysfunction in Patients with Duchenne Muscular Dystrophy: A Systematic Review
}

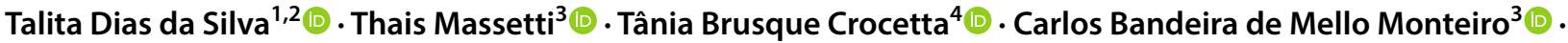 \\ Alex Carll ${ }^{2}$ - . Luiz Carlos Marques Vanderlei ${ }^{5}$ - Carlie Arbaugh $^{6} \cdot$ Fernando Rocha Oliveira $^{7}$.

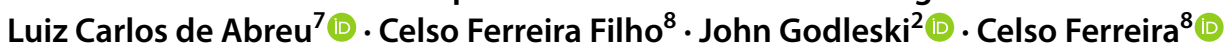

Received: 4 December 2017 / Accepted: 4 April 2018 / Published online: 25 April 2018

(c) Springer Science+Business Media, LLC, part of Springer Nature 2018

\begin{abstract}
Duchenne muscular dystrophy (DMD) is a genetic recessive disorder with progressive muscle weakness. Despite the general muscle wasting, degeneration and necrosis of cardiomyocytes have been the main causes of morbidity and death in individuals with DMD. Cardiac failure is generally preceded by disturbances in heart rate variability (HRV), and non-invasive measurement of the autonomic nervous system has been an important tool to predict adverse cardiovascular events. Hence, the application of HRV to study autonomic modulation in DMD individuals, and the establishment of correlations between HRV and heart/lung diseases, age, and mortality will have the potential to improve quality of life and life expectancy of individuals with DMD. In order to evaluate the state of the art in this field, we conducted a systematic search in Medline/ PubMed and BVS (virtual library in health) databases. We selected 8 studies using pre-defined criteria and meta-analysis revealed decreased parasympathetic activity and increased sympathetic predominance in individuals with DMD as major observations. Moreover, there is a strong association between diminished HRV and myocardial fibrosis with DMD. These patterns are evident in patients at early-stage DMD and become more prominent as disease severity and age increase. Thus, data minning clearly indicates that HRV assessment can be used as a predictor for sudden death in individuals with DMD. The use of the HRV, which is inexpensive, ubiquitously available in clinics and hospitals, and a non-invasive analysis tool, can save lives and decrease the morbity in DMD by alerting care givers to consider autonomic nervous system intervention.
\end{abstract}

Keywords Duchenne muscular dystrophy $\cdot$ Cardiomyopathy $\cdot$ Heart rate variability $\cdot$ Autonomic modulation

\begin{tabular}{|c|c|c|c|}
\hline \multicolumn{2}{|c|}{ Abbreviations } & \multirow[t]{2}{*}{ pNN50 } & \multirow{2}{*}{$\begin{array}{l}\text { Percentage of differences between RR inter- } \\
\text { vals with an absolute value greater than } 50 \mathrm{~ms}\end{array}$} \\
\hline DMD & Duchenne muscular dystrophy & & \\
\hline HRV & Heart rate variability & CVrr & Coefficient of variation of the RR interval \\
\hline BVS & Virtual library in health & $\mathrm{P}_{\mathrm{a}} \mathrm{CO}_{2}$ & Partial pressure of carbon dioxide $\left(\mathrm{CO}_{2}\right)$ in the \\
\hline Mean RR & Mean of the RR intervals & & arterial blood \\
\hline \multirow[t]{2}{*}{ RMSSD } & Square root of the mean of squared differences & LF & Low frequency \\
\hline & between successive beat intervals & $\mathrm{HF}$ & High frequency \\
\hline SDNN & Standard deviation of all normal RR interval & VLF & Very low frequency \\
\hline \multirow[t]{3}{*}{ SDANN } & Standard deviation of the means of normal-to- & ANS & Autonomic nervous system \\
\hline & normal heart periods obtained from all 5-min & BNP & Brain natriuretic peptide \\
\hline & periods throughout the whole data series & FS & Fractional shortening \\
\hline \multirow[t]{5}{*}{ SDNNi } & Average of the standard deviations of all & TTE & Trans-thoracic echocardiography \\
\hline & normal-to-normal intervals calculated from all & TD & Tissue doppler imaging \\
\hline & 5 -min periods of a $24-\mathrm{h}$ recording period & MRI & Magnetic resonance imaging \\
\hline & & $\mathrm{cMR}$ & Cardiac magnetic resonance \\
\hline & & LGE & Late gadolinium enhancement \\
\hline \multirow{3}{*}{\multicolumn{2}{|c|}{$\begin{array}{l}\text { Talita Dias da Silva } \\
\text { ft.talitadias@gmail.com }\end{array}$}} & WMA & Wall motion analysis \\
\hline & & QMT & Quantitative muscle testing \\
\hline & & ACE & Angiotensin-converting enzyme inhibitors \\
\hline
\end{tabular}


PRISMA Preferred reporting items for systematic reviews and meta-analyses

PICO Population intervention comparison outcome

SMD Standard mean differences

SD Standard deviation

\section{Introduction}

Duchenne muscular dystrophy (DMD) is a X-linked recessive disorder affecting approximately 1 in 5000 males $[1,2]$. The disease is characterized by progressive muscle wasting, which affects to a variable extent the functionality of appendicular and axial muscles. Furthermore, muscles controlling vital functions, such as respiration and cardiac movement, are frequently affected [3]. In this context, the main cause of death in DMD patients was respiratory failure, but the development of personal devices for respiratory support have significantly increased life expectancy of patients with DMD. More recently, progressive cardiac disorders are the main cause of DMD patient loss and morbity [4, 5].

Degeneration and necrosis of cardiomyocytes are among the main traits of the Duchenne syndrome. Interestingly, the majority of DMD patients are free of cardiovascular symptoms, which limits the use of conventional diagnostic methods to prevent heart failure and death of individuals. However, DMD patients often exhibit abnormalities in circadian rhythm and heart rate variability (HRV), progressing to severe congestive heart failure [4, 6-11]. Thus, assessment of cardiac electrophysiology could provide patterns of dysfunction that could forecast the course of decline [12]. Accordingly, resting heart rate is a simple measurement with important prognostic implications [13, 14]. In addition, HRV provides a well-characterized, non-invasive means to quantitatively and indirectly assess the autonomic nervous system (ANS) that controls cardiac physiology. The approach is based on fluctuations of intervals between consecutive heart beats (RR intervals) [13]. Indeed, alteration in HRV has been demonstrated to predict adverse cardiovascular events .

Since 1995, several studies have assessed HRV of individuals with DMD. Reviewing the role of HRV in DMD has considerable merit to identify common influences of sympathetic and parasympathetic branches of the ANS, pulmonary diseases, age and/or stage of the disease, influence of medication on improving autonomic modulation, and the use of HRV as a predictor of sudden death. Since heart failure has recently been identified as the leading cause of death in DMD patients, a current working hypothesis is that HRV may provide a reliable tool not only for assessing autonomic-cardiac modulation, but also for predicting risk of heart failure and mortality in DMD patients. The goal of this review is to improve our understanding of the influence of DMD on the ANS and, thereby, provide support for better therapies for individuals with DMD. Despite the relevance of this subject, there have been no meta-analyses on the relationship of DMD and HRV focusing upon the prognosis of cardiac decline. Thus, this work aims to synthesize our knowledge of autonomic modulation in individuals with DMD and establish correlations with heart diseases and progression of DMD.

\section{Method}

This review was performed according to the Preferred reporting items for systematic reviews and meta-analyses (PRISMA) guidelines, thus providing a comprehensive framework which objectively assesses indicators of quality and risk of biases of included studies [15]. The protocol for this review was not previously registered.

The scientific works were screened in the Medline/PubMed and BVS (virtual library in health) databases for the following inclusion criteria: study populations with diagnosis of DMD and analysis of heart rate variability. There were no restrictions on minimum sample size. Articles were excluded if they (1) were not data-based (e.g., books, theoretical papers, or secondary reviews), (2) were not written in the English language, (3) had populations not explicitly identified as having a diagnosis of DMD, or (4) did not include heart rate variability analysis.

All identified studies were collected in EndNote Web (Thomson Reuters) and duplicates were removed. The article search occurred in Medline/PubMed, PubMed Central, and Web of Sciences databases, through keywords that should be in all fields. We included articles that showed the terms:

"Muscular Dystrophy, Duchenne" AND "heart rate variability"

"Muscular Dystrophy, Duchenne" AND "heart rate variability" OR "autonomic nervous system"

Finally, reference lists of retrieved studies were thoroughly searched for additional relevant studies. Key words and combinations of key words were used to search the electronic databases and were organized following the Population Intervention Comparison Outcome (PICO) model. In this model, the search strategy was organized based on the topics: population (P), intervention (I), control group (C), and outcome $(\mathrm{O})$, and several searches in the aforementioned databases.

To select the articles, we used three steps as outlined in Fig. 1: (Step 1) looking for articles in databases and reading the titles and abstracts; (Step 2) exclusion of works by analysis of title, abstract, and other inclusion criteria; and (Step 3) full-text analysis of findings within eligible articles [16, 17].

After performing the initial literature searches, each study title and abstract was screened for eligibility according to 
Fig. 1 Steps used in study selection

\section{Step 1 \\ Search for articles in databases \\ Exclusion of works by predetermined criteria}

the inclusion criteria by TDS. Full texts of all potentially relevant studies were subsequently retrieved and further examined for eligibility. The PRISMA flow diagram (see Fig. 2) provides more detailed information regarding the selection process of studies.

Although the Cochrane Collaboration tool for assessing the risk of bias can be useful when studies on a review topic are numerous, given the relatively low number of papers on HRV and DMD, we believe that this tool would prohibitively diminish the number of papers in this review. We believe
Analysis of eligible studies
Fig. 2 Procedures for determination of eligibility. Adapted from Moher et al. [18]
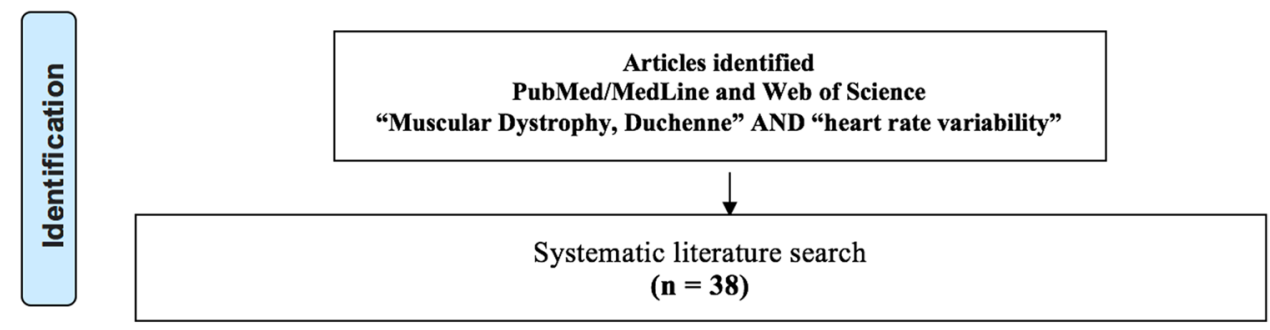

that in the future the use of this tool would be feasible in an updated review of the topic.

\section{Data Analysis}

We conducted a meta-analysis (using metafor package in $\mathrm{R}$ software, version 3.1.2) to compare "DMD" to "Control" subjects in the studies that presented absolute values of mean standard deviation of all normal RR intervals $(\mathrm{SDNN})$, square root of the mean of squared differences

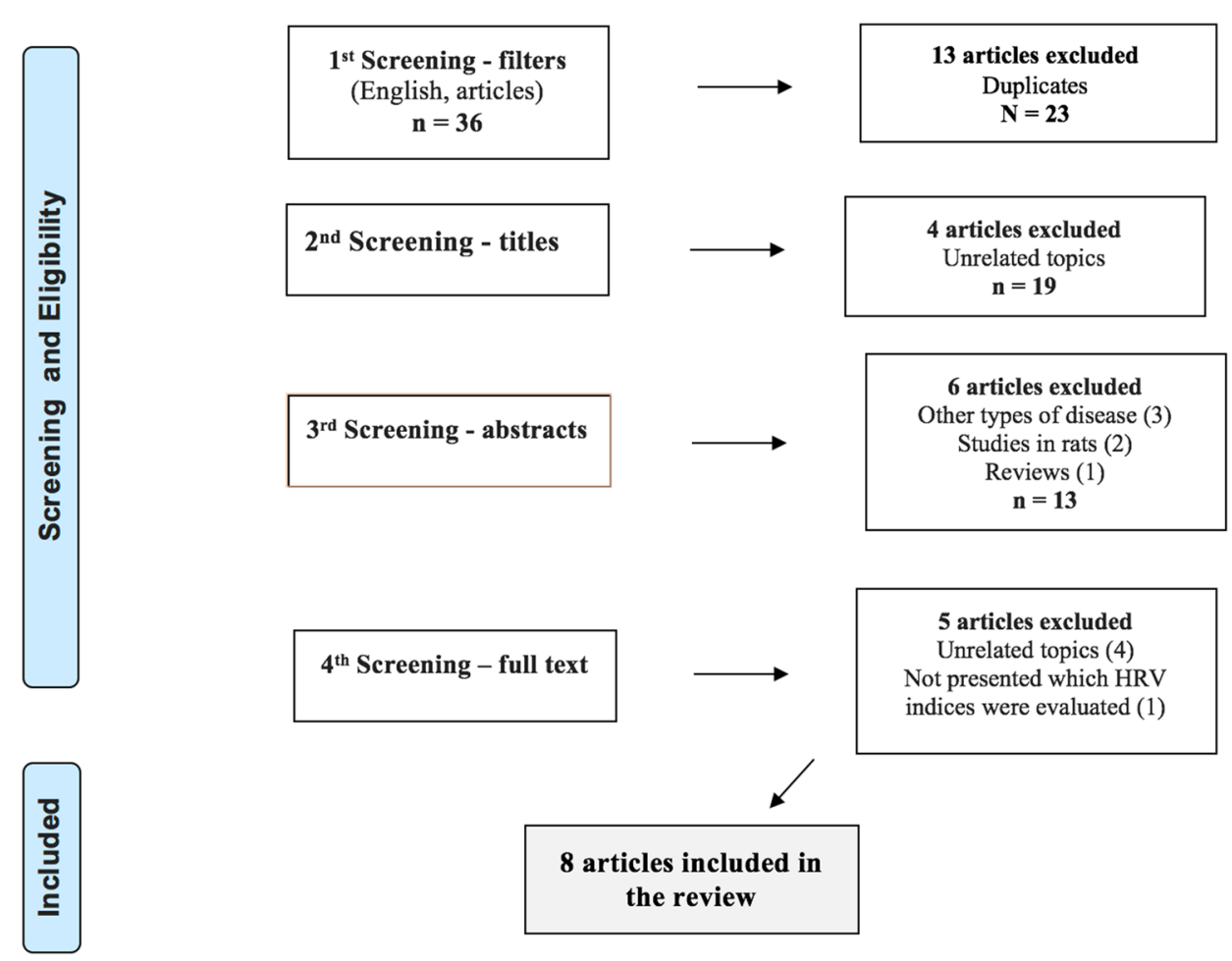


between successive beat intervals (RMSSD) and percentage of differences between RR intervals with an absolute value greater than $50 \mathrm{~ms}$ (pNN50), (mean $\pm \mathrm{SD}$ ) using "standard mean differences" (SMD) $\pm 95 \%$ confidence intervals (CI). Random effects models were used, as were appropriate tests for heterogeneity.

\section{Results}

\section{Study Selection}

Distinct databases were searched and 38 works focused on DMD and HRV were identified. Next, the 38 articles were evaluated based on the redundancy, originality, and language (English). These criterions excluded 25 out the 38 papers. Further analysis screened out five works that did not include any analysis of HRV indices. The remained 8 studies were of an empirical nature designed as a cross-sectional case control $[4,11,19,20]$, cross-sectional $[5,21]$, prospective case control cohort [19], and a prospective cohort [7] (Table 1). Together, the dataset comprises 549 DMD and 142 healthy participants, and covers distinct ethnic groups: Japanese [5, 7, 19, 22], German [21], Indian [4], Italian [11], and American [20].

The study conducted by Yotsukura et al. [19] was the first study to analyze HRV in DMD patients. They investigated whether HRV and circadian rhythm were useful in assessing autonomic dysfunction in DMD by comparing patients with different stages of DMD (mild, moderate, and severe) to normal patients. They found significant increases in relative sympathetic influence and decreases in parasympathetic modulation. The pattern was highly associated with disease progression and severity, and indicated that HRV and circadian rhythm of HRV are useful in assessing autonomic dysfunction in DMD. These findings were later validated [22] with a broader case control cohort study. Specifically, they observed progression of autonomic imbalance in DMD over a 9-year period as indicated by decreases in multiple linear HRV parameters. Specifically, the authors determined that progression of DMD was accompanied by a significant progressive reduction in LF and HF indices, denoting an increase in sympathetic tone and reduced vagal activity. The most interesting finding was that there was a significant decrease in the Mean RR, SDNN, and SDNN index in the patients with DMD who died within 6 months.

The relationship between HRV and respiratory function was examined by Lanza et al. [11] and Mochizuki et al. [5]. The study made by lanza et al. [11] showed weak correlations between forced vital capacity and HRV indices, while Mochizuki et al. [5] found that HRV index [ $\mathrm{CVrr}$ coefficient of variation of the RR interval (\%)] was negatively correlated with $\mathrm{P}_{\mathrm{a}} \mathrm{CO}_{2}$. Based on the results, the authors proposed that low HRV may indicate respiratory insufficiency in patients with DMD, since $73 \%$ of those with CVrr $<3 \%$ had hypercapnia, and $47 \%$ of them had severe hypercapnia.

Lanza et al. [11] also found no correlation between HRV and left ventricular ejection fraction. However, Thomas et al. [20] concluded that HRV decreased, manifesting as a predominance of sympathetic modulation (decreased SDANN, SDNN, RMSSD, and LF) and decreased parasympathetic modulation (decreased HF) prior to the onset of heart failure and associated with myocardial fibrosis. The authors suggest that the persistent activation of the sympathetic nervous system in DMD can be a driving force in the pathological formation of myocardial fibrosis. The authors also evaluated the effect of beta blocker use on HRV and found no significant difference on the HRV indices between DMD patients using beta blockers and DMD patients who did not; however, the authors did not appear to control for age or disease severity.

Dittrich et al. [21] evaluated Holter ECG and HRV parameters, Brain natriuretic peptide (BNP), trans-thoracic echocardiography (TTE), tissue Doppler imaging (TD), and magnetic resonance imaging (MRI) with late gadolinium enhancement (LGE) and segmental wall motion analysis (WMA) to assess diagnostic procedure efficiency of cardiac dysfunction in DMD. They concluded that MRI with segmental LGE and WMA were better than TTE and TD in exploring regional distribution and severity of myocardial damage. ECG and HRV abnormalities were common in their DMD population, but not highly predictive for segmental and global left ventricular dysfunction. Another important finding of this study was that $76 \%$ of DMD patients had altered HRV, and the authors concluded that the clinical pattern of cardiomyopathy in DMD patients aged 6-20 years was heterogeneous and not strictly age-dependent.

Inoue et al. [7] compared HRV indices with serum levels of BNP and Fractional Shortening (FS), finding that even when BNP and FS were normal, DMD patients presented with decreased SDNN. The mean heart rate at night was 71 beats/min, compared to the mean heart rate throughout the day of 57 beats/min. This heart rate difference had higher sensitivity and specificity compared to other measures in predicting abnormalities in SDNN. Also, the power of HF and pNN50 decreased with age, but LF/HF and SDNN did not vary with age. These findings again indicated HRV decreases with the severity of the disease and age.

Dhargave et al. [4] and Mochizuki et al. [5] were the only research groups to use short-term HRV measures (5 and $3 \mathrm{~min}$, respectively) in DMD patients. Both found that short-term HRV was as reliable as long-term analysis and indicated relative sympathetic dominance in DMD patients compared to controls.

The studies reviewed presented heterogeneous samples with regard to age, level of disease, and medication. Some studies encouraged patients to avoid medication for $48 \mathrm{~h}$ before 


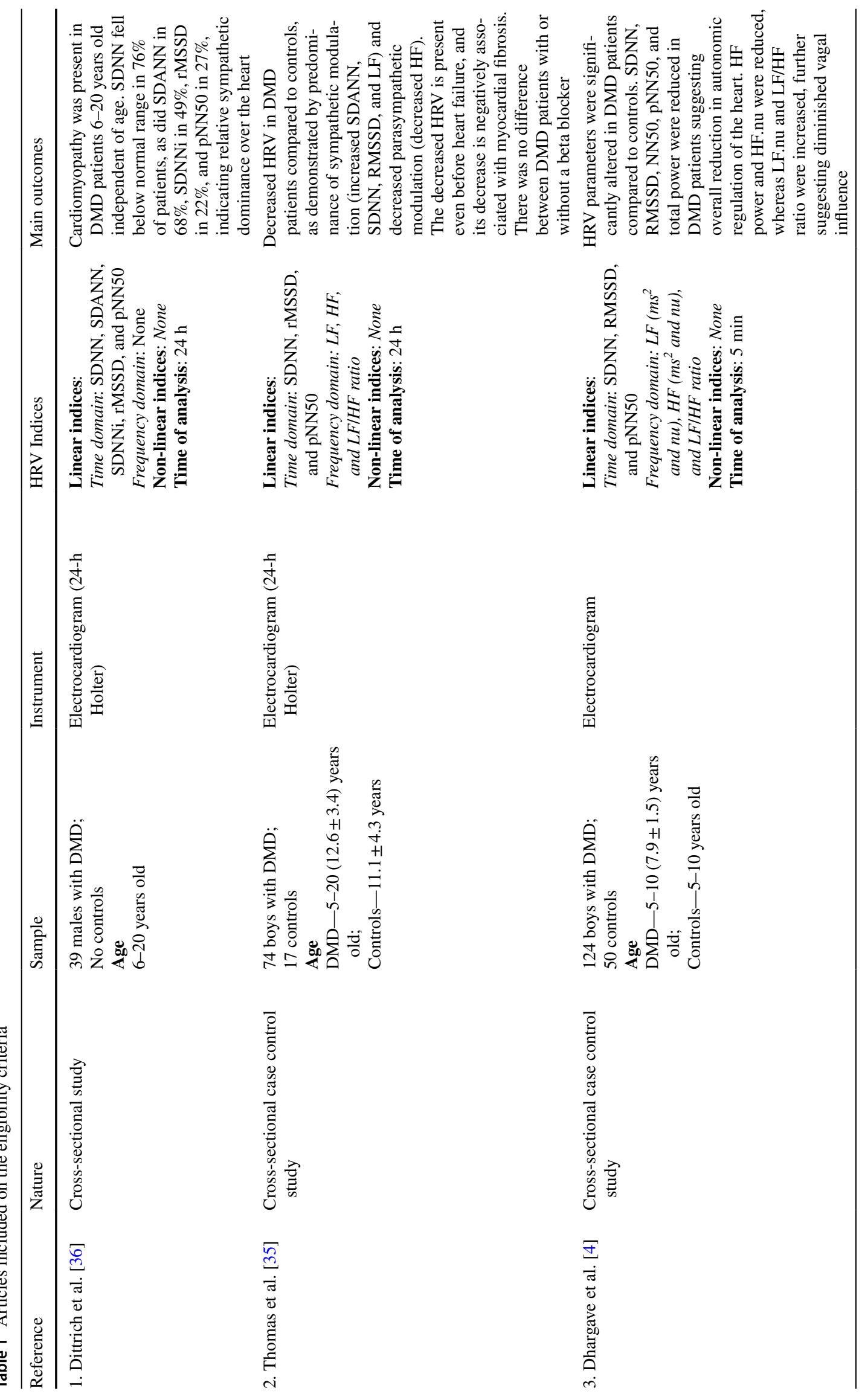




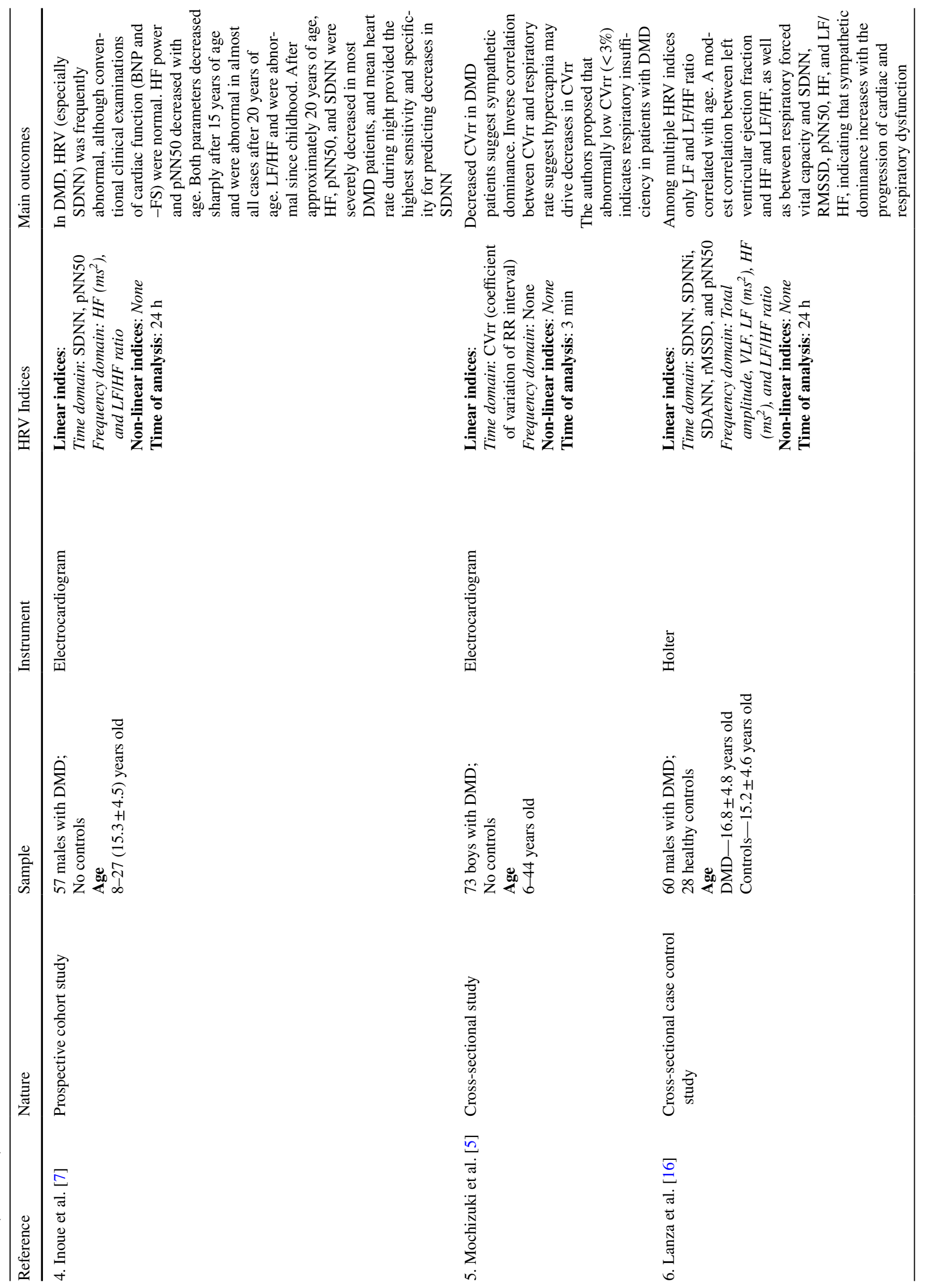




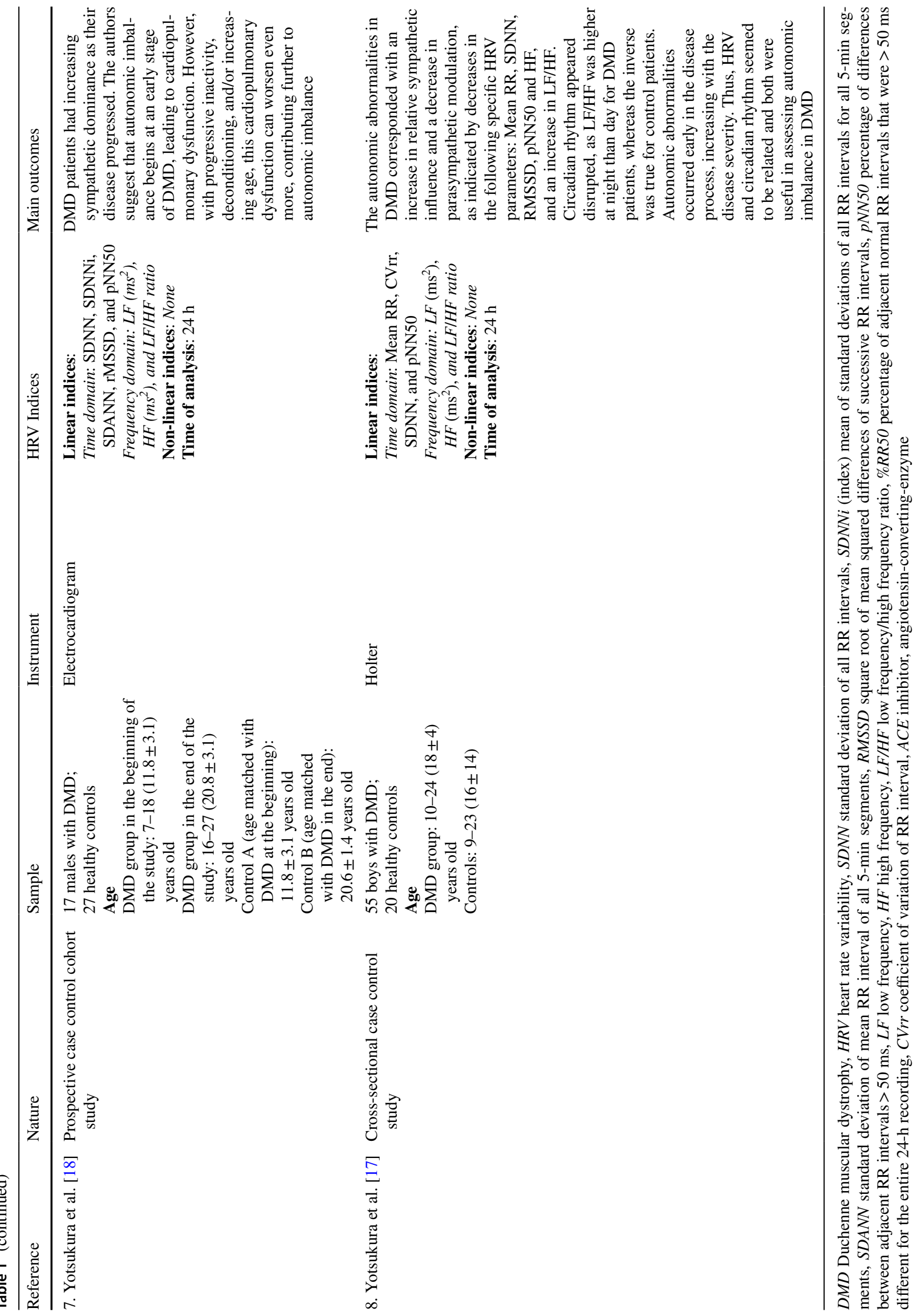


measurement $[19,22]$. In the study conducted by Inoue et al. [7], patients did not receive medication during the duration of the study. Thomas et al. [20] compared DMD patients with and without beta blockers and found no differences between the two groups.

\section{Meta-analyses-Synthesis of Results}

Meta-analyses of experimental outcomes, including the calculation of weighted mean effect sizes (Cohen's d), $95 \%$ CI, $I^{2} \%$, heterogeneity, and $p$ values from a random effects model, were assessed with the metafor package in R (http://CRAN.Rproj ect.org/package=metafor) [23]. All error bars in random forest plots are $95 \% \mathrm{CI}$; random forest plots were generated with metafor and custom R scripts. The indices SDNN, RMSSD, and pNN50 were chosen for being the indices present (with mean and standard deviation values) in the four articles with control group evaluation.

The results of standardized mean difference (SMD) and 95\% CI for each comparison are shown in Table 2.

\section{HRV Analysis}

Modulation of heart rate depends on the integration of sympathetic and parasympathetic input, and this is best reflected by short-term oscillations in beat intervals [24]. HRV analyses may be performed by several methods, including time domain, frequency domain, and non-linear analysis [10, 12, 13, 25]. However, the studies analyzed by this review only used linear indices: time and frequency domains.

\section{Time Domain}

The most common time domain indices were: RMSSD (ms), pNN50 (\%), SDNN (ms), SDANN, and SDNNi.

RMSSD is the square root of the mean of squared differences between successive beat intervals and calculated according to the following equation [26]:

$\mathrm{RMSSD}=\sqrt{\frac{\sum_{i=1}^{N-1}\left(\mathrm{RR}_{i}-\mathrm{RR}_{i+1}\right)^{2}}{N-1}}$, where $\mathrm{RRi}$ is any individual $\mathrm{RR}$ interval and $N$ is the number of RR intervals in the series of selected data. Along with pNN50, defined as the percentage of differences between RR intervals with an absolute value greater than $50 \mathrm{~ms}$, RMSSD positively correlates with relative parasympathetic dominance over cardiac rhythm. SDNN, which represents sympathetic and parasympathetic activity, is the standard deviation of the mean for all normal RR intervals expressed in milliseconds. The SDANN (ms) is the standard deviation of the means of normal-to-normal heart periods obtained from all 5-min periods throughout the whole data series, and the SDNN index (ms) is the average of the standard deviations of all normal-to-normal intervals calculated from all 5-min periods of a 24-h recording period [27-29]. A study by Mochizuki et al. [5] also reported the index CVrr, which is the coefficient of variation of RR interval (SDNN/ MeanRR*100), which is considered to reflect overall HRV (i.e., sympathetic and parasympathetic activity) [30].

\section{Frequency Domain}

The most common frequency domain indices were low frequency (LF), high frequency (HF), very low frequency (VLF), and LF/HF ratio (low frequency/high frequency ratio).

Power spectral analysis describes the periodic oscillations of the heart rate decomposed at different frequencies and amplitudes by Fast Fourier Transformation [31], with regard to oscillations in cardiovascular parameters. The HF component $(0.15-0.4 \mathrm{~Hz})$ is considered to indicate vagal influence over the heart, while the LF component $(0.04-0.15 \mathrm{~Hz})$ is believed to reflect both sympathetic and vagal influence and has been correlated with baroreflex sensitivity [25, 28]. The etiology of changes in VLF $(<0.003-0.004 \mathrm{~Hz})$ remains unclear, but VLF has been shown to predict adverse cardiac events in heart failure patients [20]. The LF/HF ratio is an index for overall sympatho-vagal balance. A ratio greater than one is an estimate of sympathetic dominance, while a $\mathrm{LF} / \mathrm{HF}$ ratio less than one is associated with parasympathetic dominance [22, 31, 32].
Table 2 Outcome measures in the meta-analysis of comparisons between all Duchenne muscular dystrophy patients and healthy control subjects in HRV measures

\begin{tabular}{llcccc}
\hline Outcome & $\begin{array}{l}\text { SMD }(95 \% \mathrm{CI}) ; \\
p \text { value }\end{array}$ & Heterogeneity & $I^{2} \%$ & $\begin{array}{l}\text { Number of } \\
\text { patients }\end{array}$ & $\begin{array}{l}\text { Number } \\
\text { of stud- } \\
\text { ies }\end{array}$ \\
\hline SDNN & $-1.70(-2.76,-0.65) ; p=0.0015$ & $<0.0001$ & 93.1 & 384 & 4 \\
RMSSD & $-0.97(-1.59,-0.36) ; p=0.0018$ & 0.0006 & 82.6 & 384 & 4 \\
pNN50 & $-1.03(-1.55,-0.51) ; p=0.0001$ & 0.0024 & 75.8 & 384 & 4 \\
\hline
\end{tabular}

$S D N N$ standard deviation of all RR intervals, $R M S S D$ square root of mean squared differences of successive RR intervals, $p N N 50$ percentage of differences between adjacent RR intervals $>50 \mathrm{~ms}$ 


\section{Time Domain Analysis (SDNN, RMSSD, pNN50)}

A total of four studies provided analyzable data for time domain analysis of HRV (SDNN, RMSSD, and pNN50) with 384 patients. A statistical difference was observed in SDNN, RMSSD, and pNN50 (Figs. 3, 4 and 5, respectively). During analysis, heterogeneity $(p<0.01)$ of the combined data was found for all time domain parameters. One study, in which only the mean values were reported, was not included in the analysis. Four studies did not include a control group, and therefore, were not included in the analysis.

For SDNN, DMD patients are estimated to present with an SDNN that is on average about one and half standard deviations below the SDNN of controls $\left(\mu^{\wedge}=-1.70\right.$ with $95 \%$ CI -2.76 to -0.65 ), but there is a considerable amount of heterogeneity in the findings (as indicated by the large estimate of $\tau^{2}=1.05$, the large $I^{2}=93.1 \%$ value, and the significant $Q(\mathrm{df}=3)=55.1438, p<0.0001)$.

Meta-analysis of SDNN effect sizes on DMD and controls is shown as a forest plot of standardized effect sizes (Cohen's d). Error bars indicate the 95\% confidence intervals of $d$. The weighted average mean effect size of all studies is represented by the central vertices of a black diamond; the outer vertices indicate the $95 \%$ confidence intervals. Control and DMD samples sizes $(n)$, mean and standard deviations SDNN (SD) of the studies are given. The $I^{2}$ of $93.1 \%$ indicates a high level of heterogeneity.

For RMSSD, similar to SDNN above, DMD patients are estimated to present with a RMSSD that is on average about one standard deviation below the RMSSD of controls $\left(\mu^{\wedge}=-0.97\right.$ with $95 \% \mathrm{CI}-1.59$ to -0.36$)$, but there is a considerable amount of heterogeneity in the findings (as indicated by the large estimate of $\tau^{2}=0.31$, the large $I^{2}=82.6 \%$ value, and the significant $Q(\mathrm{df}=3)=17.4120$, $p=0.0006)$.

Considering pNN50, similar to SDNN and RMSDD above, DMD patients are estimated to present with a pNN50 that is on average about one of the standard deviations below the pNN50 of controls $\left(\mu^{\wedge}=-1.03\right.$ with $95 \% \mathrm{CI}-1.55$ to -0.51 ), but there is a considerable amount of heterogeneity in the findings (as indicated by the large estimate of $\tau^{2}=0.21$, the large $I^{2}=75.8 \%$ value, and the significant $Q(\mathrm{df}=3)=14.3906, p=0.0024)$.

\section{Discussion}

The studies that met our meta-analysis criteria showed that, even with heterogeneity in the results, the indices evaluated (SDNN, RMSSD, and pNN50) were very significantly different in DMD patients when compared to controls. It is well

\begin{tabular}{|c|c|c|c|c|c|c|c|}
\hline Author(s) and Year & $\mathbf{n}$ & $\operatorname{SDNN}(S D)$ & $\mathbf{n}$ & SDNN(SD) & & \%Weigh & SMD $[95 \% \mathrm{Cl}]$ \\
\hline & \multicolumn{2}{|c|}{ Duchenne } & \multicolumn{3}{|c|}{ Control } & & \\
\hline Thomas et al., 2015 & 74 & $86(26.5)$ & 17 & $145(39.8)$ & $\longmapsto$ & $25.28 \%$ & $-1.99[-2.60,-1.39]$ \\
\hline Dhargave et al., 2014 & 124 & $53.4(26.5)$ & 50 & $60.6(28.9)$ & & $26.83 \%$ & $-0.26[-0.59,0.07]$ \\
\hline Lanza et al., 2001 & 60 & $94.5(28.9)$ & 28 & $164.7(44.6)$ & $\mapsto$ & $25.70 \%$ & $-2.01[-2.55,-1.47]$ \\
\hline Yotsukura et al., 1998 & 17 & $97(24)$ & 14 & $198(46)$ & $\longrightarrow$ & $22.19 \%$ & $-2.76[-3.75,-1.78]$ \\
\hline \multirow{3}{*}{\multicolumn{3}{|c|}{ Overall $(1-$ squared $=93.1 \%, p<.0001)$}} & & & & $100.00 \%$ & $-1.70[-2.76,-0.65]$ \\
\hline & & & & & $T$ & 7 & \\
\hline & & & & -4.00 & -2.00 & 0.00 & \\
\hline
\end{tabular}

Fig. 3 Meta-analysis comparing Duchenne muscular dystrophy (DMD) to "Control" subjects for studies, which presented absolute values of SDNN (mean \pm SD in $\mathrm{ms}$ ) using "Standard mean differences" (SMD) $\pm 95 \%$ confidence intervals $(\mathrm{CI})$. Effect sizes and confidence intervals are based on standardized mean differences in dis- crimination $\left(d^{\prime}\right)$. The polygon at the bottom of the panel represents the summary effect calculated using a random effects model. The square marker size indicates weight within the model. Weights are from the random effects analysis 


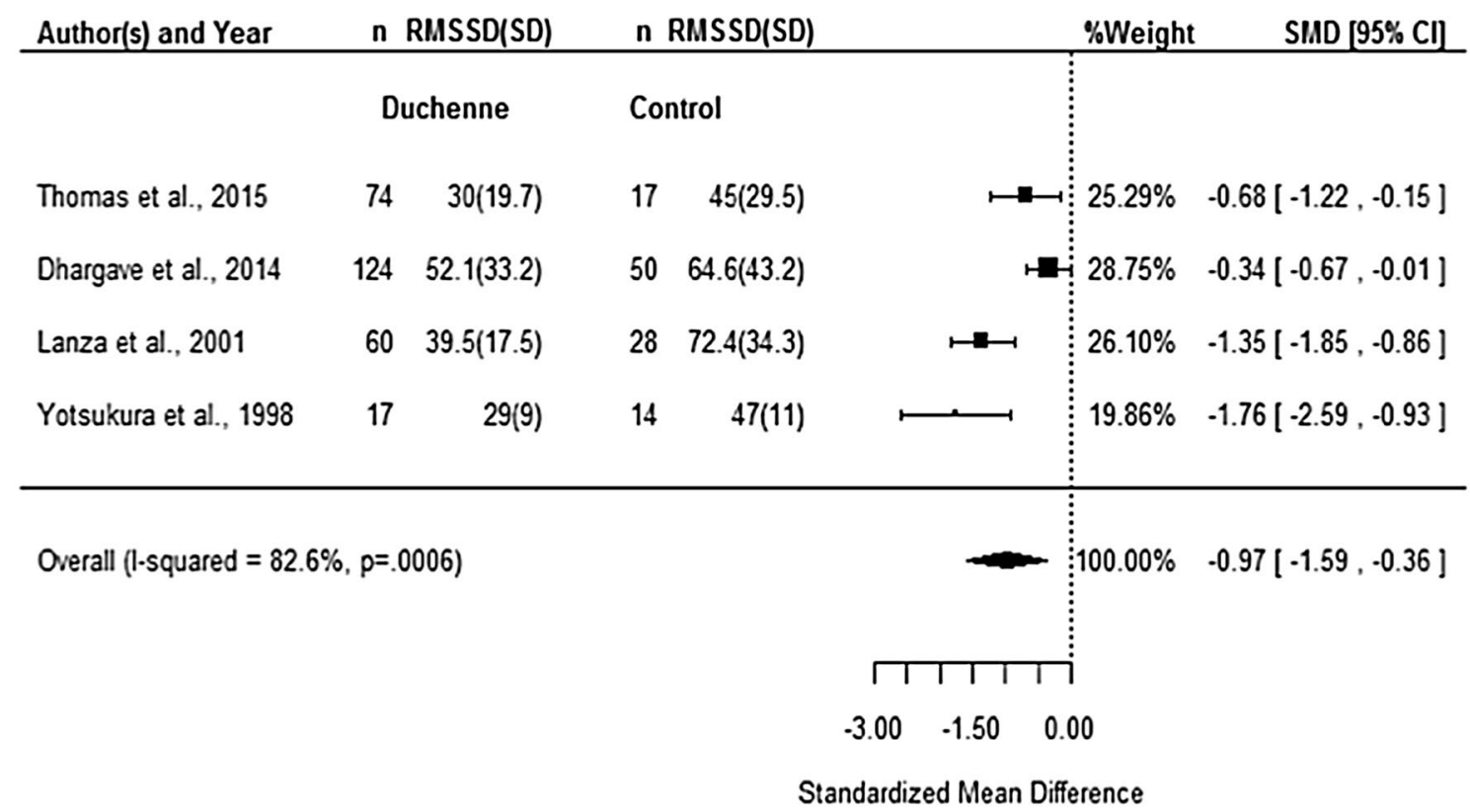

Fig. 4 Meta-analysis comparing Duchenne muscular dystrophy (DMD) to "Control" subjects for studies which presented absolute values of RMSSD (mean \pm SD in ms) using "Standard mean differences" (SMD) $\pm 95 \%$ confidence intervals (CI). Effect sizes and con- fidence intervals are based on standardized mean differences in discrimination $\left(d^{\prime}\right)$. The polygon at the bottom of panel represents the summary effect calculated using a random effects model. The square marker size indicates weight within the model

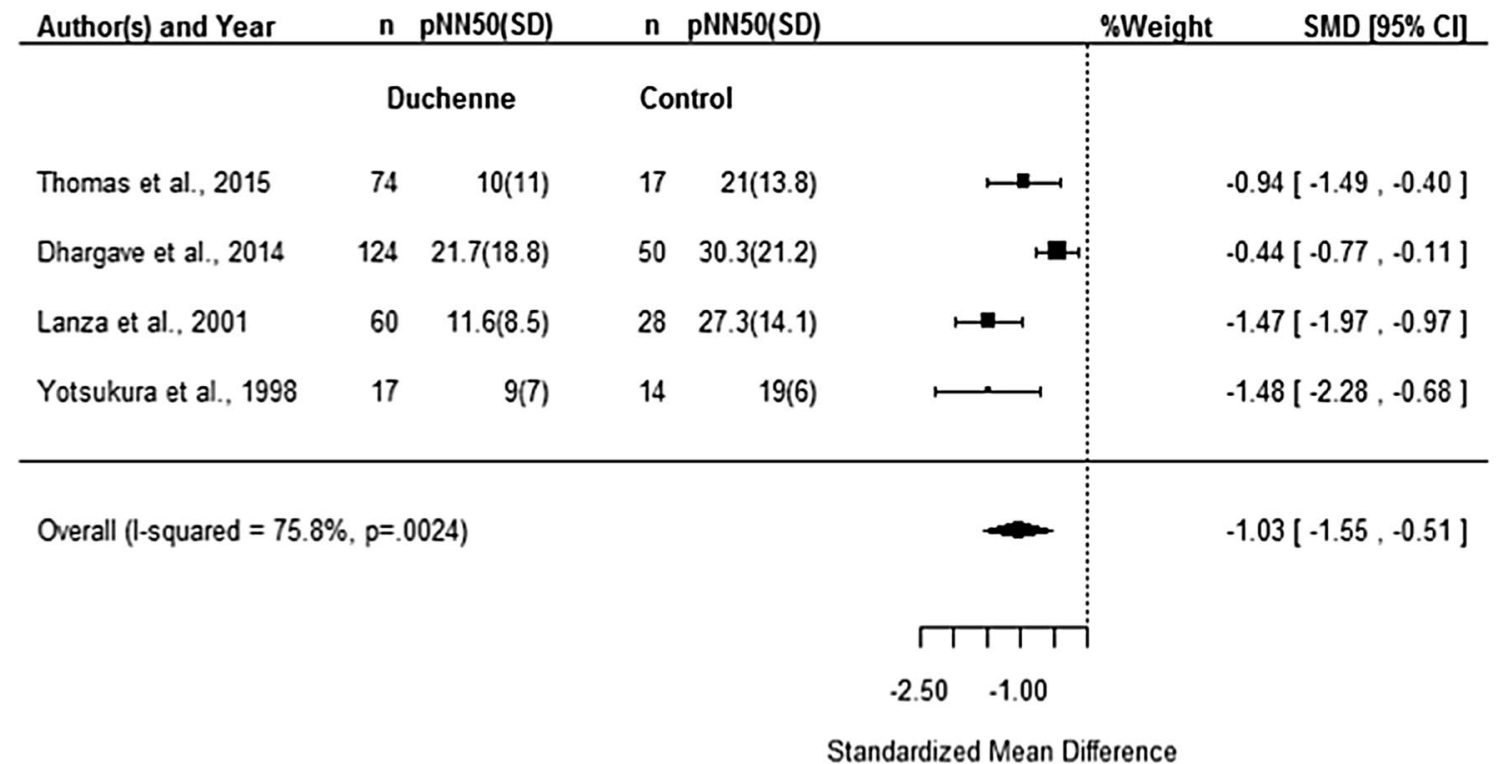

Fig. 5 Meta-analysis comparing Duchenne muscular dystrophy (DMD) to "Control" subjects for studies which presented absolute values of RMSSD (mean \pm SD in ms) using "Standard mean differences" (SMD) $\pm 95 \%$ confidence intervals $(\mathrm{CI})$. Effect sizes and con-

known that RMSSD and pNN50 are measurements that predominantly reflect parasympathetic modulation of the heart, and all studies found pNN50 to be lower in DMD patients than in controls suggesting sympathetic dominance in DMD. fidence intervals are based on standardized mean differences in discrimination $\left(d^{\prime}\right)$. The polygon at the bottom of panel represents the summary effect calculated using a random effects model. The square marker size indicates weight within the model

SDNN reflects overall variability and when lower is described as a strong predictor of death, stroke, and myocardial infarction [25]. All studies analyzed in this meta-analysis showed lower SDNN in DMD patients when compared to 
controls. We can conclude-in agreement with Inoue et al. [7] - that HRV impairment may be considered as a preclinical marker of cardiovascular dysfunction in DMD patients.

Given that heart failure has recently been identified as responsible for most of the deaths among DMD patients, it is essential to obtain an early diagnosis in order to prolong life expectancy and quality of life for these patients. As a result, we found it necessary to conduct a systematic review to characterize HRV in males with DMD, and gain a better understanding of autonomic modulation and perhaps prognosis.

All studies included in the review evaluated just linear indices, as we can see in the time domains (rMSSD, SDNN, SDNNi, SDANN, and pNN50) and frequency domains (LF, HF, and LF/HF ratio). SDNN and HF were the indices that were abnormal in the most papers reviewed $[4,7,11$, 19-22], suggesting restricted tone of the parasympathetic system for people with DMD and reduced overall heart rate variability.

Chu et al. [33] conducted a study with mice also demonstrating a preeminence of sympathetic modulation and suggesting that the etiology of cardiac muscle disease might be caused by autonomic dysfunction. However, none of the papers conducted non-linear analysis, which can better express the complex nature of heart rate variability; linear methods can not detect this as well [12].

Furthermore, the studies analyzed in this review showed restricted tone of the parasympathetic system and/or predominance of sympathetic tone in children as young as 5 years of age (Table 1 ).

\section{HRV $\times$ Age—Disease Stage}

Thomas et al. [20] showed decreased HRV in DMD patients as young as 5 years old. Yotsukura et al. [19] showed decreased HRV when compared to controls, a difference that increased with disease stage. Yotsukura et al. [22] showed that LF and HF power decreased and LF/HF ratio increased in advanced stages of DMD. All the time domain parameters were lower in DMD patients when compared to controls. These three studies demonstrate that autonomic impairment is present in patients with DMD in an early age/ disease stage, but heart failure and dyspnea (present in a late stage of the disease) seems to increase the sympathetic activity. Inoue et al. [7] also found that HF power and pNN50 decreased with age. Mochizuki et al. [5] found a correlation with $\mathrm{HR}$ and age, but no correlation with $\mathrm{CVrr}(\%)$. Lanza et al. [11] showed that HF decreased and LF/HF ratio increased with age.

Taken together, we can speculate that disregarding the presence of an autonomic imbalance at an early stage, age and disease severity highly contribute to the worsening of this imbalance.

\section{HRV and Heart Failure Induced by Myocardial Fibrosis}

The study by Thomas et al. [20] demonstrated decreased HRV in DMD patients prior to the onset of heart failure, and found an inverse correlation between HRV (SDANN) and myocardial fibrosis. The authors demonstrated that by using the association between positive Late Gadolinium Enhancement (LGE) on cMR (cardiac Magnetic Resonance) and SDANN, age, and maximal HR, the persistent activation of the sympathetic nervous system seems to be a driving force in the pathologic formation of myocardial fibrosis. In other words, HRV can be associated with heart failure and it is a useful tool to predict cardiac dysfunction that can lead to early death, thus making it possible to predict prognosis and consider treatment, aiming to increase the life expectancy of patients with DMD, as occurred after treatment for respiratory insufficiency.

In the study by Inoue et al. [7], HRV data were compared with serum levels of BNP and the SF of the left ventricle on echocardiography. Although the BNP and SF were normal, HRV parameters were frequently abnormal, which shows that the findings on the HRV indices preceded alterations in global ventricular function (SF) and neurohumoral factor (BNP) serum levels.

Posner et al. [34] investigated quantitative muscle testing (QMT) and the cardiac function (FS) in DMD patients and they found a significant relationship between skeletal muscle and cardiac function in DMD patients. The authors suggest that the cardiac function should be monitored, even when the primary outcome measures are not cardiac in nature. In order to explain the physiology of this impairment on cardiac function, in a recent study, Barbin et al. [35] examined diaphragm degeneration and cardiomyopathy in dystrophindeficient mdx mice, comparing exercised (swimming, to accelerate the diaphragm degeneration) and sedentary. They found fibrosis of the diaphragm and right and left ventricle, as well as increased density of inflammatory cells/degenerating cardiomyocytes in both the right and left ventricles with right ventricle predominance. There was also increased wall thickness of the pulmonary trunk and right ventricle which suggest pulmonary hypertension in those animals. All those parameters were increased in mice with exercise. The authors suggested that diaphragm degeneration may be the main contributor to right ventricle dystrophic pathology.

The increase in sympathetic tone may be a compensatory response to cardiac dystrophy that further drives DMD patients towards heart failure (HF); it may be the primary culprit of DMD-associated HF, or it may be a compensatory mechanism that is mostly just secondary and has little effect on progression of DMD patients towards failure. Because these patients have fibrosis, it is certainly possible that the $\mathrm{HF}$ is at least partly induced by increased adrenergic drive. 


\section{HRV and Catecholamines}

An important function of the heart is to adapt to increased needs for tissue perfusion under normal physiological conditions. This adaptation of the heart is achieved by the activation of the sympathoadrenergic system (SAS) [36]. Thus, increased sympathetic activity stimulates the adrenal medulla to produce and secrete increased amounts of adrenaline and noradrenaline, as seen in the study of Dalmaz et al. [37] in which the authors found increased amounts of urinary catecholamine, mainly in a late stage of DMD, so this increase in catecholamines seems to happen secondary to the disease and probably as a compensatory way to improve the decreased HRV.

In addition, $\mathrm{Li}$ et al. [36] stated that the SAS can persistently be activated when the heart is under stress, leading to a reduced beta-adrenergic response and it also may contribute to the increase in catecholamines. In their study with $M d x$ mice, they found that the hearts of Mdx mice partially lose beta-adrenergic responses from a young age because of myocyte loss, reduced potential for increasing myocyte contraction in $m d x$ myocytes with enhanced myocyte contractile function via molecular remodeling, and cellular desensitization in response to the hyperactive SAS.

\section{HRV and Beta Blockers}

With a timely prognosis, the question remains if drugs for regulating cardiac rate or cardiac protection improve HRV to some degree. In this regard, only Thomas et al. [20] found no treatment-related differences in HRV among those taking beta blockers (despite a clear effect on heart rate), angiotensin-converting enzyme inhibitors, or angiotensin II receptor blockers. Mochizuki et al. [5] also found no correlation between CVrr and beta blocker use in DMD patients. That is, despite reducing heart rate, beta blockers do not seem to improve HRV. It is possible that the lack of difference between HRV parameters in DMD patients with and without beta blockers can be due to DMD patients who were given beta blockers likely received it due to signs of heart failure. As such, the beta blockers may have increased their HRV to the extent that their HRV improved to be comparable to DMD patients who did not need a beta blocker.

These findings contradict what is known about beta blocker therapy: after myocardial infarction [38] and in heart failure additional beta blocker therapy has been shown to upregulate the fractal control of HRV in patients with advanced congestive heart failure [39]. Similarly, de Hartog et al. [40] found that ambient particulate matter air pollution decreased HRV in coronary artery disease patients, but beta blocker use partly inhibited this effect. They found that for people without beta blockers HRV was decreased compared to the people with beta blockers.
Perhaps beta blocker therapy does not work the same (with regard to the ANS) in people with DMD as it does in other populations. More studies are needed to better understand the underlying physiology and pharmacology.

\section{HRV and Respiratory Failure}

The etiology of HRV decreases in DMD patients remains only speculative. Mochizuki et al. [5] proposed that abnormally low CVrr (coefficient of variation of RR inter$\mathrm{val}<3 \%$ ) results from respiratory insufficiency-induced hypercapnia in patients with DMD [20]. Lanza et al. [11] found a weak but significant correlation between autonomic impairment and degree of respiratory failure. Potentially corroborating these findings, Reis et al. [41] showed that cardiac autonomic control of heart rate was associated with inspiratory muscle weakness in coronary heart failure. Nevertheless, any causality between respiratory and autonomic dysfunction remains only putative, and the two may instead derive from a common unidentified mediator. Taken together, these data suggest that impaired cardioautonomic regulation in patients with DMD likely results from multiple components, potentially including not only respiratory and mechanical cardiac dysfunction, but other factors such as structural and functional abnormalities of the sinoatrial node, neurohumoral, changes caused by inactivity of patients, and abnormal mechanoreceptor- and metaboreceptor-mediated reflexes originating from the diseased skeletal muscles [11, 22].

\section{HRV Analysis Protocol}

According to time of analysis, the majority of the studies conducted HRV analyses using Electrocardiogram or Holter for $24 \mathrm{~h}$. Dhargave et al. [4] and Mochizuki et al. [5] used 5- and 3-min analyses, respectively, but the results were consistent with previous studies which used longer (24-h) recording. Thus, it seems that short-term HRV analysis is a good tool to investigate for evidence of cardiac autonomic dysfunction in children with DMD [4]. Shortterm analysis has been used recently by several researchers $[13,14,42]$ who used a heart rate monitor for the RR interval recording and consequently HRV analysis. This method proved to be reliable and does not require patient admission to a hospital for analysis (i.e., can be done on an outpatient basis).

With 24-h analysis, Lanza et al. [11] and Inoue et al. [7] affirm that mean heart rate during night provided the highest sensitivity and specificity for predicting abnormalities of SDNN. Moreover, high frequency HRV was disproportionately diminished during the night. 


\section{HRV as a Predictor of Death}

Yotsukura et al. [19] showed that Mean RR, SDNN, and SDNNi were significantly decreased in DMD patients within 6 months prior to death. These indices should be analyzed carefully through long-term HRV measurement.

Future studies should evaluate the influence of beta blockers, angiotensin-converting enzyme inhibitors (ACE), and steroid use on improving cardiac status of DMD patients [4]. It is important to evaluate HRV in people with DMD using non-linear indices to predict and correlate heart failure and/ or muscle function, since these indices better express the complex nature of HRV [12]. Without treatment, DMD tends to culminate with fatal cardiorespiratory compromise in the early- to mid-teens [43]. The aforementioned studies suggest that HRV provides a useful and practical tool for predicting DMD exacerbation and may help reveal effective therapies by elucidating mechanisms of DMD-induced mortality.

\section{Conclusion}

A relative sympathetic dominance was evident in DMD patients from an early stage of disease, and appeared to become more prominent with an increase in disease severity and age. Although a few studies have observed correlations between reduced HRV and pulmonary function, several more have demonstrated worsening cardiac dysfunction with age and disease severity. Thus, since a primary role for autonomic imbalance in DMD is not well supported, continued study is needed to fully define this possibility. Also medication to counteract the cardiac damage of this disease remains unidentified.

Caution is needed in interpreting these results as the ChiSquare tests demonstrated large statistical heterogeneity. However, there were differences in SDNN, RMSSD, and pNN50 between DMD and controls in all studies. Moreover, HRV can be used as a preclinical marker of cardiovascular complications in DMD patients, thereby enhancing diagnosis. Lastly, HRV assessment seemed to be a predictor of sudden death through evaluation of the Mean RR, SDNN, and SDNNi indices.

Acknowledgements The authors thank CNPq (National Council for Scientific and Technological Development, process number 142280/2015-1), CAPES (Coordination for the Improvement of Higher Education Personnel, Process No. 99999.014604/2013-02) for support of this work

Author Contributions TDS, TM, FRO, and TBC participated in the acquisition of data and TDS, AC, CBMM, CA, LCA, LCMV, CFF, JG, and CF participated in the revision of the manuscript. TDS, TM, CBMM, CF determined the design, interpreted the data and all authors helped on draft the manuscript. All authors read and gave final approval for the version submitted for publication.

Data Availability The data from metanalysis of the current study are available upon request.

\section{Compliance with Ethical Standards}

Conflict of interest All authors report no conflict of interest.

Informed Consent The current study does not contain any data from individual persons.

\section{References}

1. Rodino-Klapac LR, Mendell JR, Sahenk Z (2013) Update on the treatment of Duchenne muscular dystrophy. Curr Neurol Neurosci Rep 13:332. https://doi.org/10.1007/s11910-012-0332-1

2. McNally EM (2008) Duchenne muscular dystrophy: how bad is the heart? Heart, England, pp 976-977

3. Mercuri E, Muntoni F (2013) Muscular dystrophies. Lancet 381:845-860. https://doi.org/10.1016/s0140-6736(12)61897-2

4. Dhargave P, Nalini A, Abhishekh HA, Meghana A, Nagarathna R, Raju TR, Sathyaprabha TN (2014) Assessment of cardiac autonomic function in patients with Duchenne muscular dystrophy using short term heart rate variability measures. Eur J Paediatr Neurol 18:317-320. https://doi.org/10.1016/j.ejpn.2013.12.009

5. Mochizuki H, Okahashi S, Ugawa Y, Tamura T, Suzuki M, Miyatake S, Shigeyama T, Ogata K, Kawai M (2008) Heart rate variability and hypercapnia in Duchenne muscular dystrophy. Intern Med 47:1893-1897

6. Simonds AK, Muntoni F, Heather S, Fielding S (1998) Impact of nasal ventilation on survival in hypercapnic Duchenne muscular dystrophy. Thorax 53:949-952

7. Inoue M, Mori K, Hayabuchi Y, Tatara K, Kagami S (2009) Autonomic function in patients with Duchenne muscular dystrophy. Pediatr Int 51:33-40. https://doi.org/10.1111/j.1442200X.2008.02656.X

8. Kirchmann C, Kececioglu D, Korinthenberg R, Dittrich S (2005) Echocardiographic and electrocardiographic findings of cardiomyopathy in Duchenne and Becker-Kiener muscular dystrophies. Pediatr Cardiol 26:66-72. https://doi.org/10.1007/s0024 6-004-0689-2

9. Diaz A, Bourassa MG, Guertin MC, Tardif JC (2005) Long-term prognostic value of resting heart rate in patients with suspected or proven coronary artery disease. Eur Heart J 26:967-974. https ://doi.org/10.1093/eurheartj/ehi190

10. Vanderlei LC, Pastre CM, Hoshi RA, Carvalho TD, Godoy MF (2009) Basic notions of heart rate variability and its clinical applicability. Rev Bras Cir Cardiovasc 24:205-217

11. Lanza GA, Dello Russo A, Giglio V, De Luca L, Messano L, Santini C, Ricci E, Damiani A, Fumagalli G, De Martino G, Mangiola F, Bellocci F (2001) Impairment of cardiac autonomic function in patients with Duchenne muscular dystrophy: relationship to myocardial and respiratory function. Am Heart J 141:808-812

12. Hoshi RA, Pastre CM, Vanderlei LC, Godoy MF (2013) Poincare plot indexes of heart rate variability: relationships with other nonlinear variables. Auton Neurosci 177:271-274. https://doi. org/10.1016/j.autneu.2013.05.004

13. de Carvalho TD, de Abreu LC, Mustacchi Z, Vanderlei LC, Godoy MF, Raimundo RD, Ferreira Filho C, da Silva TD, Guilhoto L, Perico V, Finotti VR, Ferreira C (2015) Cardiac 
autonomic modulation of children with Down syndrome. Pediatr Cardiol 36:344-349. https://doi.org/10.1007/s0024 6-014-1012-5

14. de Carvalho TD, Wajnsztejn R, de Abreu LC, Marques Vanderlei LC, Godoy MF, Adami F, Valenti VE, Monteiro CB, Leone C, da Cruz Martins KC, Ferreira C (2014) Analysis of cardiac autonomic modulation of children with attention deficit hyperactivity disorder. Neuropsychiatr Dis Treat 10: 613-618 https://doi. org/10.2147/ndt.s49071

15. Hutton B, Salanti G, Caldwell DM, Chaimani A, Schmid CH, Cameron C, Ioannidis JP, Straus S, Thorlund K, Jansen JP, Mulrow C, Catala-Lopez F, Gotzsche PC, Dickersin K, Boutron I, Altman DG, Moher D (2015) The PRISMA extension statement for reporting of systematic reviews incorporating network metaanalyses of health care interventions: checklist and explanations. Ann Intern Med 162:777-784. https://doi.org/10.7326/m14-2385

16. de Menezes LDC, Massetti T, Oliveira FR, de Abreu LC, Malheiros SRP, Trevizan IL, Moriyama CH, de Mello Monteiro CB (2015) Motor learning and virtual reality in down syndrome; a literature review. Int Archiv Med 8:1-11

17. Massetti T, Crocetta TB, Silva TDD, Trevizan IL, Arab C, Caromano FA, Monteiro CBM (2017) Application and outcomes of therapy combining transcranial direct current stimulation and virtual reality: a systematic review. Disabil Rehabil Assist Technol 12:551-559. https://doi.org/10.1080/17483107.2016.1230152

18. Moher D, Liberati A, Tetzlaff J, Altman DG (2010) Preferred reporting items for systematic reviews and meta-analyses: the PRISMA statement. Int J Surg 8:336-441. https://doi. org/10.1016/j.ijsu.2010.02.007

19. Yotsukura M, Sasaki K, Kachi E, Sasaki A, Ishihara T, Ishikawa K (1995) Circadian rhythm and variability of heart rate in Duchenne-type progressive muscular dystrophy. Am J Cardiol 76:947-951

20. Thomas TO, Jefferies JL, Lorts A, Anderson JB, Gao Z, Benson DW, Hor KN, Cripe LH, Urbina EM (2015) Autonomic dysfunction: a driving force for myocardial fibrosis in young Duchenne muscular dystrophy patients? Pediatr Cardiol 36:561-568. https ://doi.org/10.1007/s00246-014-1050-z

21. Dittrich S, Tuerk M, Haaker G, Greim V, Buchholz A, Burkhardt B, Fujak A, Trollmann R, Schmid A, Schroeder R (2015) Cardiomyopathy in Duchenne muscular dystrophy: current value of clinical, electrophysiological and imaging findings in children and teenagers. Klin Padiatr 227:225-231. https://doi. org/10.1055/s-0034-1398689

22. Yotsukura M, Fujii K, Katayama A, Tomono Y, Ando H, Sakata K, Ishihara T, Ishikawa K (1998) Nine-year follow-up study of heart rate variability in patients with Duchenne-type progressive muscular dystrophy. Am Heart J 136:289-296. https://doi. org/10.1053/hj.1998.v136.89737

23. Viechtbauer W, Smits L, Kotz D, Bude L, Spigt M, Serroyen J, Crutzen R (2015) A simple formula for the calculation of sample size in pilot studies. J Clin Epidemiol 68:1375-1379. https://doi. org/10.1016/j.jclinepi.2015.04.014

24. Zamuner AR, Cunha AB, da Silva E, Negri AP, Tudella E, Moreno MA (2011) The influence of motor impairment on autonomic heart rate modulation among children with cerebral palsy. Res Dev Disabil 32:217-221. https://doi.org/10.1016/j. ridd.2010.09.020

25. Cygankiewicz I, Zareba W (2013) Heart rate variability. Handb Clin Neurol 117:379-393. https://doi.org/10.1016/b978-0-44453491-0\$400031-6

26. Marães VR, Catai AM, Milan LA, Rojas FA, Oliveira LD, Teixeira LC, Gallo Junior L, Silva ED (2003) Determinação e validação do limiar de anaerobiose a partir de métodos de análise de frequência cardíaca e de sua variabilidade. Rev Soc Cardiol Estado de Säo Paulo 13: 1-16
27. Pumprla J, Howorka K, Groves D, Chester M, Nolan J (2002) Functional assessment of heart rate variability: physiological basis and practical applications. Int J Cardiol 84:1-14

28. Arab C, Dias DP, Barbosa RT, Carvalho TD, Valenti VE, Crocetta TB, Ferreira M, Abreu LC, Ferreira C (2016) Heart rate variability measure in breast cancer patients and survivors: a systematic review. Psychoneuroendocrinology 68:57-68. https://doi. org/10.1016/j.psyneuen.2016.02.018

29. Aubert AE, Seps B, Beckers F (2003) Heart rate variability in athletes. Sports Med 33:889-919

30. Kadoya M, Koyama H, Kurajoh M, Kanzaki A, Kakutani-Hatayama M, Okazaki H, Shoji T, Moriwaki Y, Yamamoto T, Emoto M, Inaba M, Namba M (2015) Sleep, cardiac autonomic function, and carotid atherosclerosis in patients with cardiovascular risks: HSCAA study. Atherosclerosis 238:409-414. https://doi. org/10.1016/j.atherosclerosis.2014.12.032

31. Yperzeele L, van Hooff RJ, De Smedt A, Nagels G, Hubloue I, De Keyser J, Brouns R (2016) Feasibility, reliability and predictive value of in-ambulance heart rate variability registration. PLoS ONE 11:e0154834. https://doi.org/10.1371/journal.pone.0154834

32. La Rovere MT, Bigger JT Jr, Marcus FI, Mortara A, Schwartz PJ (1998) Baroreflex sensitivity and heart-rate variability in prediction of total cardiac mortality after myocardial infarction. ATRAMI (Autonomic Tone and Reflexes After Myocardial Infarction) Investigators. Lancet 351:478-484

33. Chu V, Otero JM, Lopez O, Sullivan MF, Morgan JP, Amende I, Hampton TG (2002) Electrocardiographic findings in mdx mice: a cardiac phenotype of Duchenne muscular dystrophy. Muscle Nerve 26:513-519. https://doi.org/10.1002/mus.10223

34. Posner AD, Soslow JH, Burnette WB, Bian A, Shintani A, Sawyer DB, Markham LW (2016) The correlation of skeletal and cardiac muscle dysfunction in Duchenne muscular dystrophy. J Neuromuscul Dis 3:91-99. https://doi.org/10.3233/jnd-150132

35. Barbin IC, Pereira JA, Bersan Rovere M, de Oliveira Moreira D, Marques MJ, Santo Neto H (2016) Diaphragm degeneration and cardiac structure in mdx mouse: potential clinical implications for Duchenne muscular dystrophy. J Anat 228:784-791. https://doi. org/10.1111/joa.12443

36. Li Y, Zhang S, Zhang X, Li J, Ai X, Zhang L, Yu D, Ge S, Peng Y, Chen X (2014) Blunted cardiac beta-adrenergic response as an early indication of cardiac dysfunction in Duchenne muscular dystrophy. Cardiovasc Res 103:60-71. https://doi.org/10.1093/ cvr/cvu 119

37. Dalmaz Y, Peyrin L, Mamelle JC, Tuil D, Gilly R, Cier JF (1979) The pattern of urinary catecholamines and their metabolites in Duchenne myopathy, in relation to disease evolution. J Neural Transm 46:17-34 DOI

38. Lampert R, Ickovics JR, Viscoli CJ, Horwitz RI, Lee FA (2003) Effects of propranolol on recovery of heart rate variability following acute myocardial infarction and relation to outcome in the Beta-Blocker Heart Attack Trial. Am J Cardiol 91:137-142

39. Li RW, Levi DM, Klein SA (2004) Perceptual learning improves efficiency by re-tuning the decision 'template' for position discrimination. Nat Neurosci 7:178-183. https://doi.org/10.1038/ nn1183

40. de Hartog JJ, Lanki T, Timonen KL, Hoek G, Janssen NA, IbaldMulli A, Peters A, Heinrich J, Tarkiainen TH, van Grieken R, van Wijnen JH, Brunekreef B, Pekkanen J (2009) Associations between PM2.5 and heart rate variability are modified by particle composition and beta-blocker use in patients with coronary heart disease. Environ Health Perspect 117:105-111. https://doi. org/10.1289/ehp.11062

41. Reis MS, Arena R, Archiza B, de Toledo CF, Catai AM, BorghiSilva A (2014) Deep breathing heart rate variability is associated with inspiratory muscle weakness in chronic heart failure. Physiother Res Int 19:16-24. https://doi.org/10.1002/pri.1552 
42. Raimundo RD, de Abreu LC, Adami F, Vanderlei FM, de Carvalho TD, Moreno IL, Pereira VX, Valenti VE, Sato MA (2013) Heart rate variability in stroke patients submitted to an acute bout of aerobic exercise. Transl Stroke Res 4:488-499. https:// doi.org/10.1007/s12975-013-0263-4

43. Bushby K, Finkel R, Birnkrant DJ, Case LE, Clemens PR, Cripe L, Kaul A, Kinnett K, McDonald C, Pandya S, Poysky J, Shapiro
F, Tomezsko J, Constantin C (2010) Diagnosis and management of Duchenne muscular dystrophy, part 1: diagnosis, and pharmacological and psychosocial management. Lancet Neurol 9:77-93. https://doi.org/10.1016/s1474-4422(09)70271-6

\section{Affiliations}

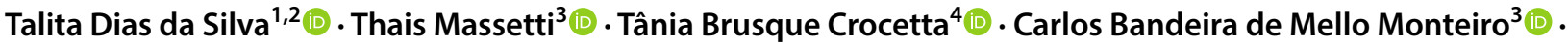 Alex Carll $^{2}$ (1) - Luiz Carlos Marques Vanderlei ${ }^{5}$ (D) Carlie Arbaugh $^{6} \cdot$ Fernando Rocha Oliveira $^{7}$.

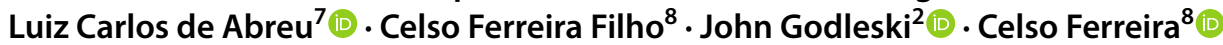

Thais Massetti

thaismassetti@gmail.com

Tânia Brusque Crocetta

taniabrusque@gmail.com

Carlos Bandeira de Mello Monteiro carlosmonteiro@usp.br

Alex Carll

alex.carll@louisville.edu

Luiz Carlos Marques Vanderlei

lcmvanderlei@fct.unesp.br

Carlie Arbaugh

cja62@cornell.edu

Fernando Rocha Oliveira

oliveira.fernando.rocha@hotmail.com

Luiz Carlos de Abreu

luizcarlos@usp.br

Celso Ferreira Filho

dr.celso@uol.com.br

John Godleski

jgodlesk@hsph.harvard.edu

Celso Ferreira

doutorcelsoferreira@gmail.com
1 Paulista School of Medicine, Federal University of São Paulo, Rua Napoleão de Barros, 715, Vila Clementino, São Paulo, SP CEP: 04024-003, Brazil

2 Department of Environmental Health, Harvard TH Chan School of Public Health, 677 Huntington Ave, Boston, MA 02115, USA

3 Graduate Program in Rehabilitation Sciences, Faculty of Medicine, University of São Paulo, Rua Cipotânea, 51, São Paulo, SP 05360-000, Brazil

4 Faculty of Medicine of ABC, Avenida Príncipe de Gales, 821, Santo André, SP 09060-950, Brazil

5 Sao Paulo State University - UNESP, Rua Roberto Símonsen, 305, Presidente Prudente, SP 19060-900, Brazil

6 Stanford University School of Medicine, 450 Serra Mall, Stanford, CA 94305, USA

7 School of Public Health, University of São Paulo, Avenida Dr. Arnaldo, 715, São Paulo, SP 01246-904, Brazil

8 Graduate Program in Medicine (Cardiology), Paulista School of Medicine, Federal University of São Paulo, Rua Napoleão de Barros, 715, São Paulo, SP 04024-003, Brazil 